\title{
A light-hole exciton in a quantum dot
}

\author{
Y. H. Huo ${ }^{1 \star}$, B. J. Witek ${ }^{2 \star}$, S. Kumar', J. R. Cardenas ${ }^{3}$, J. X. Zhang', N. Akopian ${ }^{2,4}$, R. Singh ${ }^{3}$, E. Zallo1, \\ R. Grifone ${ }^{5}$, D. Kriegner ${ }^{5}$, R. Trotta ${ }^{1,5}$, F. Ding ${ }^{1}$, J. Stangl ${ }^{5}$, V. Zwiller ${ }^{2}$, G. Bester ${ }^{3 \star}$, A. Rastelli ${ }^{1,5 \star}$ \\ and O. G. Schmidtt,
}

\begin{abstract}
A light-hole exciton is a quasiparticle formed from a single electron bound to a single light hole. This type of fundamental excitation, if confined inside a semiconductor quantum dot, could be advantageous in quantum information science and technology. However, it has been difficult to access it so far, because confinement and strain in conventional quantum dots favour a ground-state single-particle hole with a predominantly heavy-hole character. Here we demonstrate the creation of a light-hole exciton ground state by applying elastic stress to an initially unstrained quantum dot. Its signature is clearly distinct from that of the well-known heavy-hole exciton and consists of three orthogonally polarized bright optical transitions and a fine-structure splitting of hundreds of microelectronvolts between in-plane and out-of-plane components. This work paves the way for the exploration of the fundamental properties and of the potential relevance of three-dimensionally confined light-hole states in quantum technologies.
\end{abstract}

pitaxial semiconductor quantum dots are considered as candidate building blocks for quantum technologies, as they can act both as hosts of static quantum bits (excitons $s^{1,2}$ or spins $s^{3-8}$ ) or as triggered sources of single and entangled photons ${ }^{9-11}$. In particular, quantum dots can confine carriers with a spin coherence time longer than in the corresponding bulk materials. Hole spins, especially, are receiving increasing attention because their limited hyperfine interaction with the nuclear spin bath should reduce decoherence compared with electron spins ${ }^{6-8}$. All experimental studies presented so far have dealt with heavy holes. This is because quantum confinement lifts the valence band degeneracy and leaves heavy-hole states energetically well above the light-hole states. Further energetic separation is provided by the compressive strain, which is required for the growth of self-assembled quantum dots such as InGaAs islands in a GaAs matrix. Some proposals suggest, however, that using light holes instead of heavy holes would be beneficial for quantum information technologies. These include the coherent conversion of photons into electron spins ${ }^{12}$, the possibility to directly control the light-hole spin state using microwaves ${ }^{13}$, the direct tomographic measurement of the electron spin state or spin coherence $e^{14}$, and the faster and more stable control of a magnetic impurity spin coupled to a quantum $\operatorname{dot}^{15}$. For the realization of these and future proposals, the light hole should be the ground state, as any decay channel would reduce the coherence time ${ }^{16}$. Whereas quantum-dot light-hole excitons involving an excited hole state have been studied ${ }^{17}$, and light-hole-heavy-hole mixing induced by shape and/or strain anisotropy has often been observed in the ground state of conventional II-VI and III-V quantum dots ${ }^{18,19}$, reports on systems with a light-hole ground state are limited to nanostructures with a large height/base ratio ${ }^{20-22}$ resembling vertical nanorods. Owing to the broad linewidth of the exciton emission from the dot-in-dot studied in ref. 22 and the absence of single-nanostructure data for the columnar quantum dots studied in refs 20,21, it remains, however, unclear whether a geometrical route is appropriate to obtain quantum dots with a light-hole ground state and with optical properties comparable to state-of-the-art self-assembled quantum dots.

Here we thus address two open questions: can we make high-quality quantum dots with a ground state of dominant light-hole-type? What does the excitonic emission of such a quantum dot look like?

Our successful answer to the first question is to use strain engineering rather than a complete redesign of the quantum-dot geometry: starting from almost unstrained GaAs quantum dots in an AlGaAs matrix with a conventional heavy-hole ground state, we reverse the energetic order of the heavy-hole and light-hole bands by biaxial tensile stress. The process preserves the zero-dimensional character of the exciton, as demonstrated by photon antibunching measurements, reported in Supplementary Section I.4.

Our quantum dots are obtained by local droplet etching of nanoholes into an AlGaAs surface followed by GaAs-filling and $\mathrm{AlGaAs}$ overgrowth ${ }^{23}$. A sketch of the heterostructure is shown in Fig. 1a. Compared with strained self-assembled quantum dots, GaAs/AlGaAs quantum dots grown on $\mathrm{GaAs}(001)$ substrates are almost unstrained and their height, which influences the light-hole-heavy-hole splitting, can be controlled by adjusting the amount of GaAs used to fill the nanoholes. As in-plane anisotropy of the confinement potential is expected to contribute to light-hole-heavy-hole mixing, the growth protocol is further optimized to obtain highly symmetric quantum $\operatorname{dots}^{23}$ (Fig. 1b). Finally, to allow the light hole to become the ground state, a biaxial tensile strain of about $0.36 \%$ is induced on the $\sim 8-\mathrm{nm}$ high quantum dots by embedding them into symmetrically prestressed membranes, which are then released from the substrate ${ }^{24}$ (see arrows in Fig. 1a,c. Details are given in Methods and Supplementary Sections I.1 and I.2). Further fine-tuning of the

\footnotetext{
${ }^{1}$ Institute for Integrative Nanosciences, IFW Dresden, Helmholtzstraße 20, 01069 Dresden, Germany, ${ }^{2}$ Kavli Institute of Nanoscience, Delft University of Technology, Lorentzweg 1, 2628CJ Delft, The Netherlands, ${ }^{3}$ Max-Planck-Institute for Solid State Research, Heisenbergstraße 1, 70569 Stuttgart, Germany, ${ }^{4}$ Department of Applied Physics, Eindhoven University of Technology, 5600 MB Eindhoven, The Netherlands, ${ }^{5}$ Institute of Semiconductor and Solid State Physics, Johannes Kepler University Linz, Altenbergerstraße 69, 4040 Linz, Austria, ${ }^{6}$ Center for Advancing Electronics Dresden (CfAED), Dresden University of Technology, 01067 Dresden, Germany. *e-mail:y.huo@ifw-dresden.de; B.J.Witek@tudelft.nl; g.bester@fkf.mpg.de; armando.rastelli@jku.at
} 


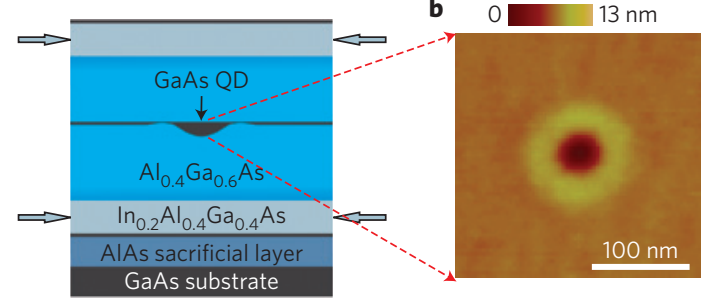

c
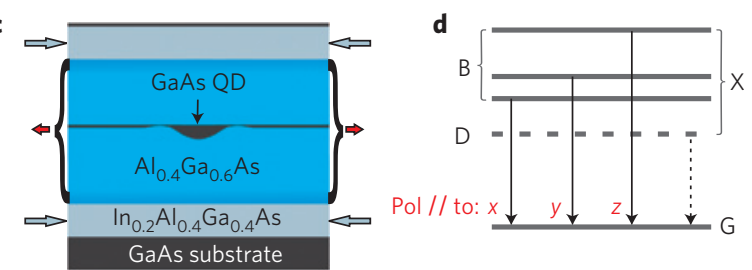

Figure 1 | A quantum dot with a light-hole exciton ground state. a, Schematics of the GaAs quantum-dot (QD) heterostructure grown on $\mathrm{GaAs}(001)$ substrate. The GaAs quantum-dot layer is in the middle of a pre-stressed (In)AIGaAs membrane. The length of the horizontal arrows is proportional to the magnitude of in-plane strain in the layers. $\mathbf{b}$, Atomic force microscopy (AFM) image of a representative droplet-etched nanohole on the AIGaAs surface before GaAs filling. c, Schematics of the GaAs quantum-dot heterostructure after selective etching of the AIAs sacrificial layer followed by strain relaxation and bond back. The initially unstrained quantum dot is now tensile strained. $\mathbf{d}$, Energy level diagram and allowed dipole transitions with corresponding polarization directions. $X$ indicates the neutral light-hole exciton, which consists of a dark (D) state and three bright (B) states decaying to the crystal ground state (G). $x$ and $y$ denote in-plane crystal directions [110] and [11̄0] and $z$ denotes the growth direction [001]. Pol, polarization.

biaxial strain is achieved by placing the membranes onto a piezoelectric actuator ${ }^{25,26}$.

To experimentally discriminate a light-hole exciton from a heavy-hole exciton, we consider how a light-hole, with spin projection $J_{z}= \pm 1 / 2$ along the [001] crystal direction $(z)$, manifests itself in the polarization state of emitted photons (Supplementary Section II for details on what follows). We constructed basis states for a four-dimensional pure light-hole exciton space composed of the electron spin ( $\uparrow$ and $\downarrow)$ and light-hole spin ( and $\Downarrow)$ :

$$
|1\rangle=|\uparrow \uparrow\rangle,|2\rangle=|\downarrow \Downarrow\rangle,|3\rangle=|\uparrow \Downarrow\rangle,|4\rangle=|\downarrow \uparrow\rangle
$$

The first two basis states $(|1\rangle$ and $|2\rangle)$ with parallel spins have an angular momentum projection of $J_{1, z}=+1$ and $J_{2, z}=-1$, and emit circularly polarized photons ( $\sigma^{+}$and $\sigma^{-}$respectively). The two remaining states $(|3\rangle$ and $|4\rangle)$ with antiparallel spins both have $J_{3, z}=J_{4, z}=0$ giving rise to linearly polarized photons in the $z$ growth direction. However, these are not yet the eigenstates in a quantum dot, where electron-hole exchange interactions should be considered. We thus derived the exchange interaction Hamiltonian from the theory of invariants ${ }^{27}$ and solved it for a pure light-hole exciton. By assuming that the momentum matrix elements are isotropic, we find the polarization of the four excitonic eigenstates as shown in Fig. 1d (see also ref. 28). The exciton (X) consists of three optically bright (B) states and one dark (D) state. Two bright states, $\mathrm{B}_{x}$ and $\mathrm{B}_{y}$, are linear superpositions of the basis states $|1\rangle$ and $|2\rangle$. The resulting polarization of $B_{x}(|1\rangle+|2\rangle)$ and $B_{y}(|1\rangle-|2\rangle)$ is linear along the [110] $(x)$ and [110] $(y)$ crystal directions. The symmetric linear superposition of states $|3\rangle$ and $|4\rangle$ forms another bright state $\mathrm{B}_{z}(|3\rangle+|4\rangle)$ polarized along the $z$ direction. Finally, the oscillator strength of the antisymmetric superposition $|3\rangle-|4\rangle$ cancels out and a dark state $\mathrm{D}_{z}$ is formed. This means that, in contrast to the conventional heavy-hole exciton configurations, which include two dark states, an additional bright emission line should be observable by collecting the luminescence perpendicular to the growth direction.

To verify whether the induced tensile strain is sufficient to switch the light hole to the ground state of the valence band of our quantum dots, we excite micro-photoluminescence with a laser beam focused on the sample (001) surface and collect linearpolarization-resolved photoluminescence spectra of as-grown and tensile strained quantum dots along both $z$ and $x$ (cleaved edge) directions. The spectra for a representative as-grown quantum dot are shown in Fig. 2a,b and are fully consistent with a heavy-hole exciton: two in-plane polarized bright-exciton states, $B_{1}$ and $B_{2}$, separated by a fine-structure splitting (FSS) of $\sim 10 \mu \mathrm{eV}$. (Generally the directions 1 and 2 do not coincide with the crystal directions $x$ and $y$ in highly symmetric quantum $\operatorname{dots}^{23}$. However, for quantum dots close to a cleaved edge, we observed that the polarization direction of one of the lines of the in-plane polarized doublet is roughly aligned with the cleaved edge ( $y$ direction) and that the FSS and polarization anisotropy are slightly larger than for quantum dots a few micrometres away from the edge. A possible explanation is discussed in Supplementary Section I.3.b.) After membrane undercut, the average quantum-dot emission energy redshifts by about $50 \mathrm{meV}$ owing to the tensile biaxial strain, as illustrated by the spectra from another representative quantum dot, shown in Fig. 2c,d. Most importantly, all quantum-dot spectra measured so far exhibit a new line located at $\sim 430 \mu \mathrm{eV}$ above the in-plane polarized doublet $\mathrm{B}_{1,2}$, see Supplementary Section I.3.d. This line, indicated as $\mathrm{B}_{z}$ in Fig. 1d, is barely visible when observed along the growth direction, but is very pronounced when detected from the cleaved edge of the sample and is linearly polarized along $z$ (Fig. 2d). In addition, its intensity exhibits the same excitation-power dependence as $B_{1,2}$ and photon cross-correlation measurements between $B_{z}$ and $B_{1,2}$ show a lack of coincidence events at zero delay, see Supplementary Sections I.3.f and I.4. All of these observations are in line with our expectations for a light-hole exciton having three bright recombination channels: $\mathrm{B}_{1,2}$ and $\mathrm{B}_{z}$. (The origin of the relatively weak $z$-polarized emission observed in Figs $2 \mathrm{~b}$,d and $3 b$ in correspondence to $B_{1,2}$ is discussed in Supplementary Section I.3.b.)

To better resolve the $B_{1,2}$ lines and to access also the dark state $\mathrm{D}_{z}$ of a light-hole exciton, we carried out magnetic-fielddependent micro-photoluminescence measurements in the Faraday configuration, that is, with the magnetic field parallel to the $z$ direction $^{29,30}$. In fact, different from the heavy-hole case in the Faraday configuration, the $\mathrm{D}_{z}$ line is expected to become visible owing to magnetic-field-induced mixing with the $\mathrm{B}_{z}$ line. This is analogous to the more common situation encountered for the dark states of a heavy-hole exciton, which become partially bright when a magnetic field is applied in the $x-y$ plane (Voigt configuration) owing to mixing with the $x-y$-polarized bright excitons. (A quantitative description of the behaviour of a light-hole exciton in magnetic field is beyond the scope of this work and will be presented elsewhere.) Figure 3 a shows polarization-resolved spectra of a single quantum dot collected along the $z$ direction. The $B_{1}$ and $B_{2}$ lines, which are initially linearly polarized and split by $9.5 \mu \mathrm{eV}$ (see bottom of Fig. 3a), exhibit a diamagnetic shift and split further owing to the Zeeman effect. When the Zeeman splitting becomes larger than the exchange splitting, the exchange interaction for states $B_{1}$ and $B_{2}$ can be neglected and we observe the recovery of the circular polarization of the basis states $|1\rangle$ and $|2\rangle$. The picture changes in Fig. $3 \mathrm{~b}$, where light is collected along the $x$ direction. Besides $\mathrm{B}_{z}$, the initially dark state $\mathrm{D}_{z}$ appears as a $z$-polarized line as the field is increased. 

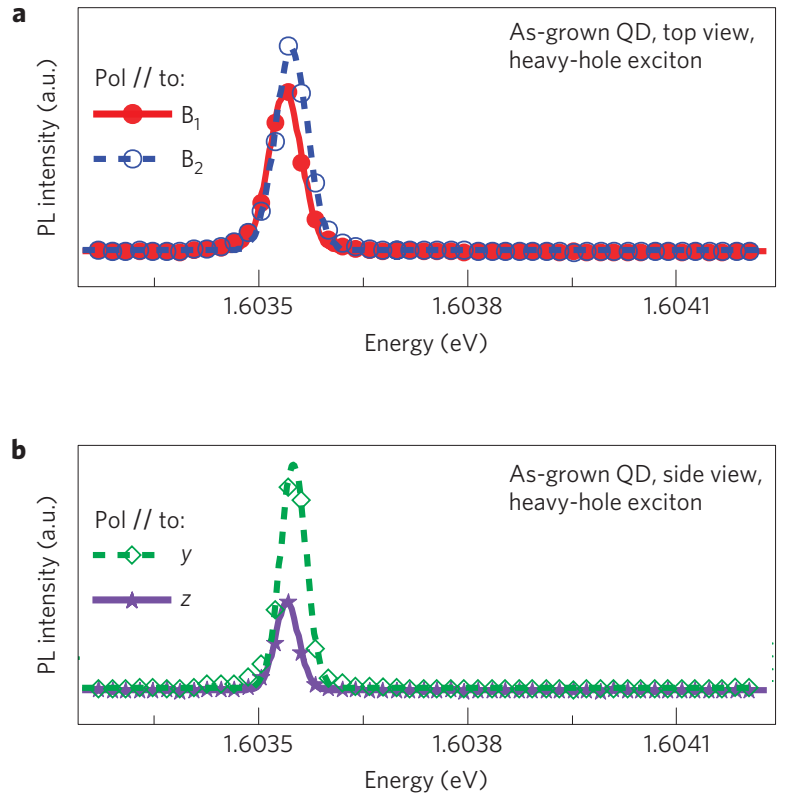
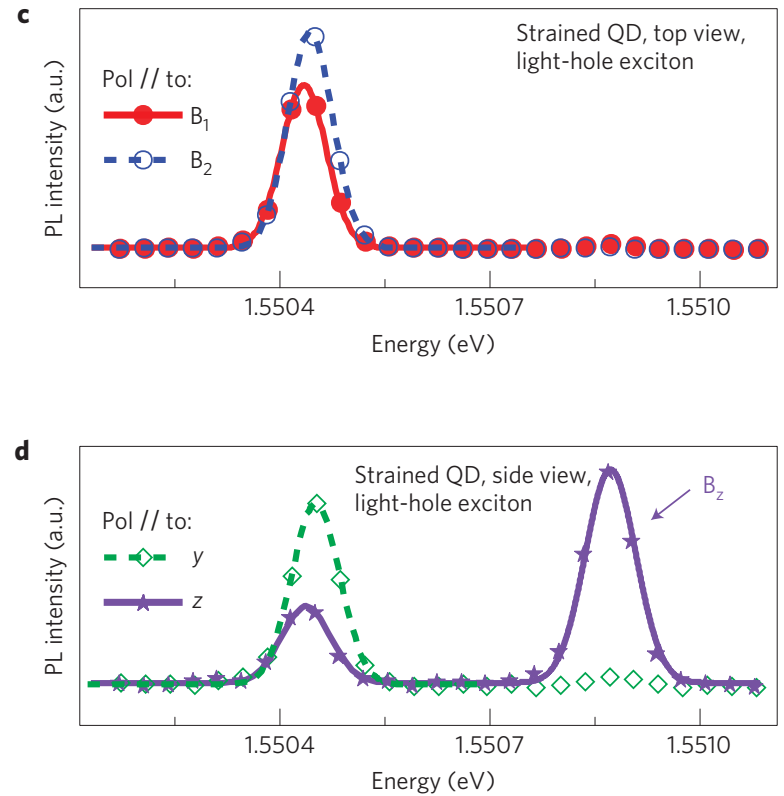

Figure 2 | Representative micro-photoluminescence spectra of heavy-hole and light-hole excitons in single GaAs/AIGaAs quantum dots. a-d, Linearly polarized spectra of neutral excitons along two perpendicular directions for one representative as-grown quantum dot $(\mathbf{a}, \mathbf{b})$ and one tensile-strained quantum dot (c,d). PL, photoluminescence. Spectra are collected along the conventional $z$ [001] direction in a,c and along the $x$ [110] direction from a cleaved edge in $\mathbf{b}, \mathbf{d}$. In $\mathbf{a}, \mathbf{c}$ filled and open circles correspond to the in-plane polarized lines $B_{1}$ and $B_{2}$. For the two quantum dots, the $B_{1}$ line has polarization direction close to $x$. In $\mathbf{b}, \mathbf{d}$ open diamonds correspond to light polarized along the cleaved edge (y[110] direction), with spectra dominated by the emission of $B_{2}$, and stars correspond to light polarized along the $z$ [001] growth direction. Besides the projection of the in-plane components (mostly $B_{1}$ ) in the $z$ direction (Supplementary Section I.3.b), the spectrum of a light-hole exciton in $\mathbf{d}$ is dominated by the $z$-polarized line $B_{z}$. In all plots, lines are Lorentzian fits of the experimental data.

Analogous to the in-plane polarized lines $\mathrm{B}_{1}$ and $\mathrm{B}_{2}$, the lines $\mathrm{B}_{z}$ and $\mathrm{D}_{z}$, initially split by the exchange interaction, shift and split owing to the diamagnetic and Zeeman effect. By fitting the peak energy positions of $\mathrm{B}_{z}$ and $\mathrm{D}_{z}$, we find that $\mathrm{D}_{z}$ lies $482 \mu \mathrm{eV}$ below the energy of $\mathrm{B}_{z}$ at zero field. Note that the exchange splitting between $\mathrm{B}_{z}$ and $\mathrm{D}_{z}$ is so large that it remains dominant over the Zeeman splitting up to the largest magnetic fields available. As a consequence, the basis states $|3\rangle$ and $|4\rangle$ cannot be restored. However, a brightening of $\mathrm{D}_{z}$ is clear and all of the four light-hole exciton states are now observed. Similar behaviour and values of the $\mathrm{D}_{z}-\mathrm{B}_{z}$ splitting have been observed for all measured quantum dots (Supplementary Section I.3.e).

Although the experimental results discussed so far are in line with the qualitative expectations of the mesoscopic model, a quantitative prediction of the fine-structure is necessary to assert that the unexpectedly large FSS is indeed a feature of a single light-hole exciton state. We have thus performed numerical calculations using the atomistic empirical pseudopotential method and configuration interaction, including the electron-hole exchange interaction ${ }^{31,32}$. The structure for the simulation was constructed from the measured quantum-dot morphology by directly using atomic force microscopy (AFM) topographs of representative AlGaAs nanoholes before and after GaAs filling ${ }^{23,33}$ (see Supplementary Fig. 1). Owing to the lack of compressive strain and the relatively large height $(\sim 8 \mathrm{~nm})$, the splitting between the dominant heavy-hole and dominant light-hole states is significantly reduced, compared with the common InGaAs/GaAs case. To simulate the experimental conditions, we introduce an in-plane tensile strain to the simulation cell and relax the atomic positions as well as the simulation cell in the $z$ direction $^{31}$. In Fig. 4 a we plot the heavy-hole and light-hole characters of the valence-band ground state, $\mathrm{h}_{0}$, which is obtained from the projection of the atomistic quantum-dot wavefunctions onto Bloch states of the underlying bulk, as a function of the induced strain (Supplementary Section II.4). We see that, already for a moderate tensile strain value of $\sim 0.2 \%$, the character shifts from dominantly heavy-hole to dominantly light-hole. For the experimentally determined strain value of $0.36 \%$ we expect that $h_{0}$ has $\sim 95 \%$ light-hole character. Furthermore, the measured value of the in-plane polarization anisotropy for the strained quantum dot in Fig. 2c,d hints at a very large (99\%) light-hole character, following the model of ref. 34 (Supplementary Section I.6). In Fig. $4 \mathrm{~b}$ we plot the excitonic fine structure as a function of the tensile strain. The energies are given relative to the lowest (dark) exciton state $\mathrm{D}_{z}$. At zero strain we have the well-known situation where the two bright states are polarized in-plane and are split by a FSS of $\sim 8 \mu \mathrm{eV}$, in good agreement with the experimental values, whereas the dark states are nearly degenerate. When tensile strain is introduced, the situation changes markedly with one $z$-polarized bright state between 200 and $600 \mu \mathrm{eV}$ above the $\mathrm{D}_{z}$, and two bright states, split by only a few microelectronvolts and polarized in-plane, at an energy of around $30-40 \mu \mathrm{eV}$ above $\mathrm{D}_{z}$. These results are in excellent quantitative agreement with the experimental data, demonstrating that the quantum dots presented here can be used as model systems to study and possibly make use of light-hole states.

According to the calculation in Fig. 4b, the energy separation $\Delta E$ between $\mathrm{B}_{z}$ and the in-plane polarized doublet monotonically increases with increasing tensile strain. To test this prediction, we have transferred pre-stressed membranes onto a piezoelectric substrate using gold-thermo-compression bonding ${ }^{35}$ (Fig. 5, inset), allowing us to increase (decrease) the tensile strain by simply decreasing (increasing) the electric field applied across the piezo. (For this experiment, another sample with asymmetric quantum $\operatorname{dots}^{23}$ was used, see Supplementary Sec. I.1.a for details. For such quantum dots, the $\mathrm{B}_{z}$-line becomes easily visible also when observed along the $z$ direction, indicating a radiating dipole that is tilted 
a
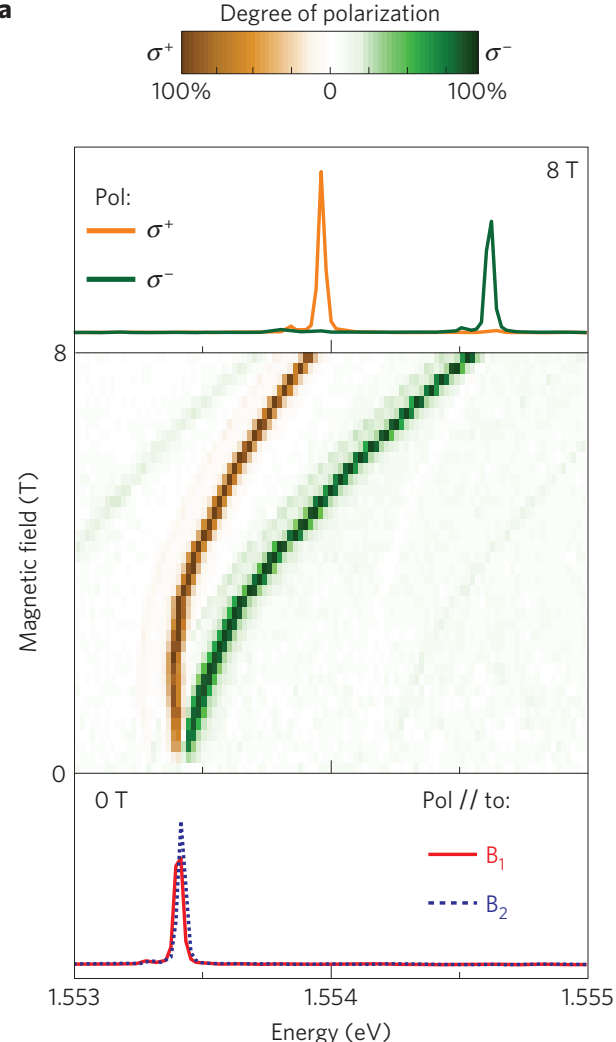
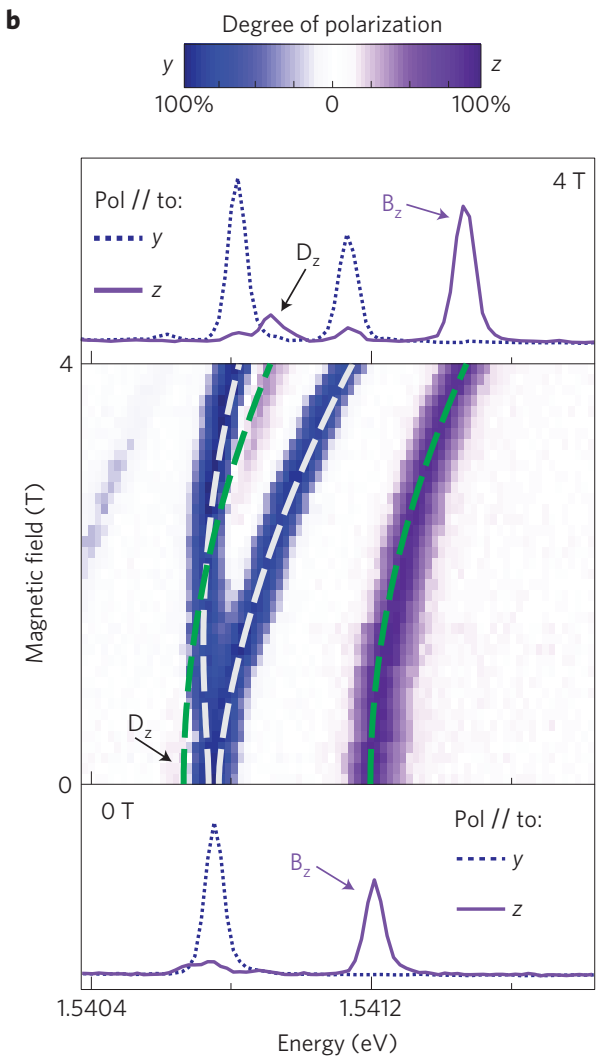

Figure 3 | Representative polarization-resolved micro-photoluminescence spectra of light-hole excitons in a magnetic field applied along the growth direction (Faraday configuration). $\mathbf{a}, \mathbf{b}$, Spectra for light collected along the $z$ direction (a) and along the $x$ direction (b). The colour plots in $\mathbf{a}, \mathbf{b}$ are obtained by superimposing the intensities of circularly polarized spectra (with $\sigma^{+}$and $\sigma^{-}$polarization) and linearly polarized spectra (with polarization parallel to the $y$ and $z$ directions), respectively. The polarization-resolved spectra shown on the top and bottom of $\mathbf{a}$ and $\mathbf{b}$ correspond to zero and maximum magnetic field. The dashed lines in the colour plot in $\mathbf{b}$ represent fits of the peak positions for the in-plane polarized $B_{1}$ and $B_{2}$ lines (white) and $z$-polarized $B_{z}$ and $D_{z}$ lines (green). $D_{z}$ appears as the magnetic field is increased, as shown in the top spectrum of $\mathbf{b}$.
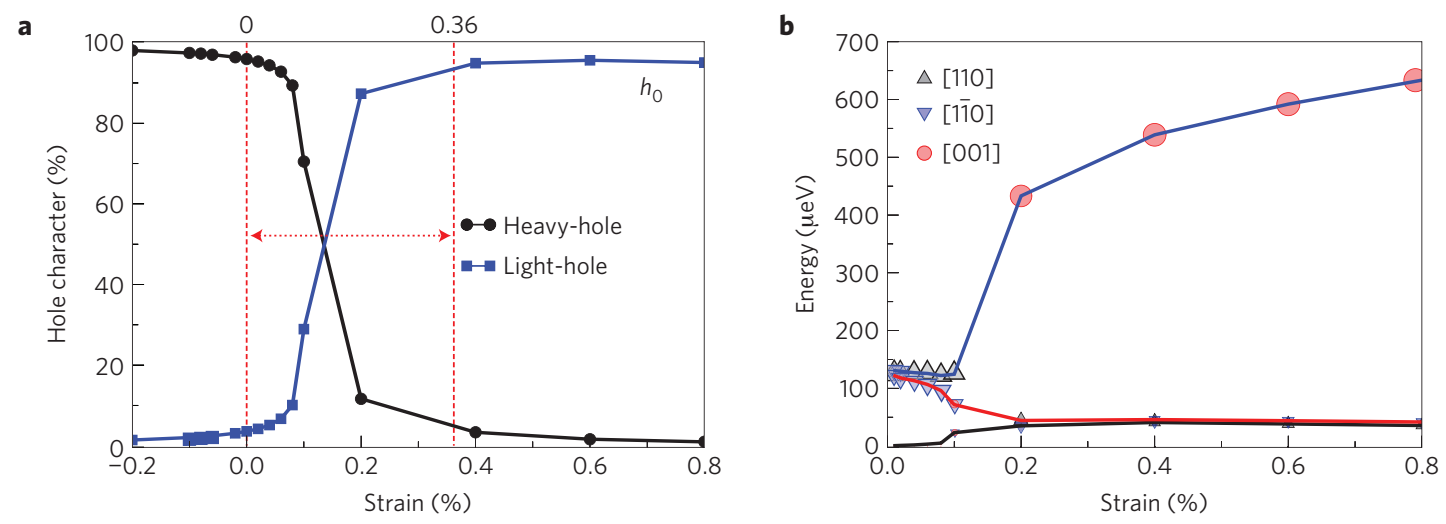

Figure 4 | Calculation results using the experimental quantum-dot structure. a, Analysis of the hole character of the ground state $h_{0}$ as a function of in-plane biaxial strain. The used GaAs quantum dot has a shape constructed from representative AFM topographs (see Supplementary Fig. 1). The dashed lines and horizontal arrow indicate the value of strain before and after membrane undercut. $\mathbf{b}$, Excitonic fine structure, setting the energy of the lowest exciton state to zero. The black and blue triangles represent the transitions polarized in the growth plane (001); the red circles represent the transitions polarized along the growth direction [001]. The size of the symbols is proportional to the oscillator strengths. The lowest exciton transition energies are between 1.56 and $1.45 \mathrm{eV}$ for the range of used strains.

away from the ideal $z$ direction. This facilitates the strain-dependent measurements by allowing photoluminescence collection along the $z$ direction.) As shown in Fig. 5, when the electric field changes from 23.3 to $-10 \mathrm{kV} \mathrm{cm}^{-1}$ the exciton energy decreases by about $9 \mathrm{meV}$ and $\Delta E$ increases by about $44 \mu \mathrm{eV}(474-518 \mu \mathrm{eV})$, see Supplementary Fig. 16 for the corresponding spectra. The calculations predict an increase in $\Delta E$, when going from 0.2 to $0.4 \%$ strain, of $105 \mu \mathrm{eV}(388-493 \mu \mathrm{eV})$ accompanied by a reduction in exciton energy of $31 \mathrm{meV}(1.550-1.519 \mathrm{eV})$. Therefore, the change in $\Delta E$ relative to the change in emission energy is $\sim 3.4 \mu \mathrm{eV} \mathrm{meV}{ }^{-1}$, in good agreement with the experimental result of $\sim 4.9 \mu \mathrm{eV} \mathrm{meV}^{-1}$. 


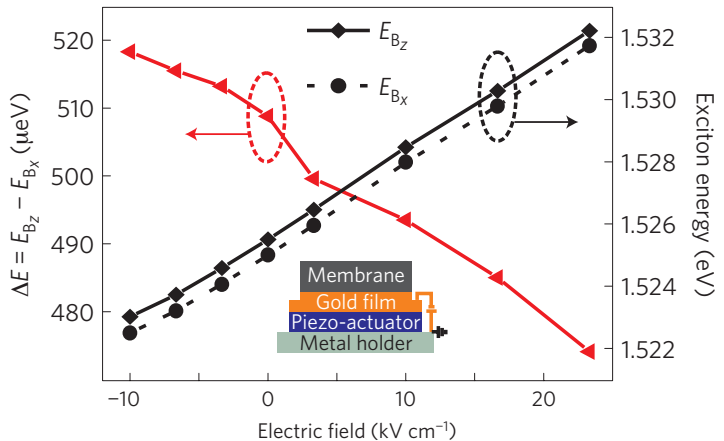

Figure 5 | Strain tuning of a light-hole exciton. Exciton emission energy and energy splitting $(\Delta E)$ between the $B_{z}$ line and the low-energy component of the in-plane polarized doublet (here indicated as $B_{x}$ ) measured using an asymmetric quantum dot (Supplementary Section I.1.a) in a membrane that was released from the GaAs substrate and placed onto a piezoelectric actuator. The in-plane tensile strain increases for decreasing electric fields applied to the actuator. The inset is a sketch of the experimental configuration.

In conclusion, we have shown that the excitonic ground state of self-assembled GaAs quantum dots can be switched from the common dominant heavy-hole to the light-hole type by releasing pre-stressed membranes with initially unstrained quantum dots. The excitonic fine structure was investigated both experimentally and theoretically. The high optical quality of the presented dots (full-width at half-maximum $\sim 23 \mu \mathrm{eV}$ for as-grown quantum $\operatorname{dots}^{23}$, full-width at half-maximum $\sim 38 \mu \mathrm{eV}$ for lighthole quantum dots in released membranes, see Supplementary Section I.3.g), the compatibility of membrane processing with electrical control ${ }^{35}$, and the fact that the ground hole state can have more than $95 \%$ light-hole character for tensile strains of $\sim 0.4 \%$ demonstrate that three-dimensionally confined light-holes may soon be explored as new semiconductor-based quantum systems for quantum communication technologies.

\section{Methods}

Sample growth, processing and structural characterization. The samples studied here were grown by molecular beam epitaxy on semi-insulating $\operatorname{GaAs}(001)$ substrates. Eight-nanometre-deep nanoholes were obtained by depositing 0.5 monolayers of excess $\mathrm{Al}$ on an $\mathrm{Al}_{0.4} \mathrm{Ga}_{0.6} \mathrm{As}(001)$ surface at a substrate temperature of $600{ }^{\circ} \mathrm{C}$ followed by $5 \mathrm{~min}$ annealing under $\mathrm{As}_{2}$ flux. The nanoholes were then overgrown with $2 \mathrm{~nm}$ GaAs (quantum-dot material), followed by 2 min annealing favouring hole filling, $37 \mathrm{~nm} \mathrm{Al}_{0.4} \mathrm{Ga}_{0.6} \mathrm{As}$, and $7 \mathrm{~nm}$ graded $\mathrm{Al}_{x} \mathrm{Ga}_{1-x} \mathrm{As}$ (with $x$ varying from 0.4 to 0.44 ). The morphology of the quantum dots was obtained by measuring with AFM the surface of two additional samples, where the growth was interrupted after nanohole etching and after GaAs overgrowth. The quantum-dot layer was placed into symmetrically pre-stressed membranes, which include one $\mathrm{In}_{0.2} \mathrm{Al}_{0.4} \mathrm{Ga}_{0.4}$ As stressor layer below and another above the active structure. By etching a sacrificial AlAs layer placed below the membrane structure, the strain, which was originally confined in the InAlGaAs layers, is shared with the initially unstrained heterostructure (see horizontal arrows in Fig. la,c, with lengths proportional to the strain magnitudes). After etching, the membranes bond-back to the underlying substrate. A tensile strain of $0.36 \%$ was induced in the quantum-dot layer according to X-ray diffraction measurements.

Optical characterization. We performed micro-photoluminescence measurements using a $532 \mathrm{~nm}$ continuous-wave frequency-doubled $\mathrm{Nd} \mathrm{YVO}_{4}$ laser focused onto the sample surface using a microscope objective with a numerical aperture of 0.42 . The same objective was used to collect light along the $z$ [001] direction. A second objective was mounted at $90^{\circ}$ to collect light emitted along the $x$ [110] direction. The membranes with underlying substrate were cleaved and mounted on the cold-finger of a cryostat equipped with multiple optical windows. For polarization analysis, we rotated an achromatic $\lambda / 2$ waveplate by $360^{\circ}$ at $2^{\circ}$ steps in front of a linear polarizer. For the magnetic-field-dependent photoluminescence measurements, the samples were mounted on the top and side facets of a cubic sample holder. A vector magnet was employed to measure each sample in the
Faraday geometry with magnetic fields up to $B_{\max }=8 \mathrm{~T}\left(\mathrm{~B}_{\max }=4 \mathrm{~T}\right)$ along the growth direction for samples on the top (side) facet.

Received 7 January 2013; accepted 3 October 2013; published online 17 November 2013

\section{References}

1. Zrenner, A. et al. Coherent properties of a two-level system based on a quantum-dot photodiode. Nature 418, 612-614 (2002).

2. Ramsay, A. J. A review of the coherent optical control of the exciton and spin states of semiconductor quantum dots. Semicond. Sci. Technol. 25, 103001 (2010).

3. Greilich, A. et al. Mode locking of electron spin coherences in singly charged quantum dots. Science 313, 341-345 (2006).

4. Press, D., Ladd, T. D., Zhang, B. \& Yamamoto, Y. Complete quantum control of a single quantum dot spin using ultrafast optical pulses. Nature 456, 218-221 (2008).

5. Nowack, K. C., Koppens, F. H. L., Nazarov, Y. V. \& Vandersypen, L. M. K. Coherent control of a single electron spin with electric fields. Science 318, 1430-1433 (2007).

6. Laurent, S. et al. Electrical control of hole spin relaxation in charge tunable InAs/GaAs quantum dots. Phys. Rev. Lett. 94, 147401 (2005).

7. Gerardot, B. D. et al. Optical pumping of a single hole spin in a quantum dot. Nature 451, 441-444 (2008).

8. Greve, K. D. et al. Ultrafast coherent control and suppressed nuclear feedback of a single quantum dot hole qubit. Nature Phys. 7, 872-878 (2011).

9. Michler, P. et al. A quantum dot single-photon turnstile device. Science 290, 2282-2285 (2000).

10. Akopian, N. et al. Entangled photon pairs from semiconductor quantum dots. Phys. Rev. Lett. 96, 130501 (2006).

11. Salter, C. L. et al. An entangled-light-emitting diode. Nature 465, 594-597 (2010).

12. Vrijen, R. \& Yablonovitch, E. A spin-coherent semiconductor photo-detector for quantum communication. Physica E 10, 569-575 (2001).

13. Sleiter, D. \& Brinkman, W. F. Using holes in GaAs as qubits: An estimate of the Rabi frequency in the presence of an external rf field. Phys. Rev. B 74, 153312 (2006).

14. Kosaka, H. et al. Spin state tomography of optically injected electrons in a semiconductor. Nature 457, 702-705 (2009).

15. Reiter, D. E., Kuhn, T. \& Axt, V. M. Coherent control of a single Mn spin in a quantum dot via optical manipulation of the light hole exciton. Phys. Rev. B 83, $155322(2011)$

16. Schmidt, K. H., Medeiros-Ribeiro, G., Oestreich, M., Petroff, P. M. \& Döhler, G. H. Carrier relaxation and electronic structure in InAs self-assembled quantum dots. Phys. Rev. B 54, 11346-11353 (1996).

17. Karlsson, K. F. et al. Fine structure of exciton complexes in high-symmetry quantum dots: Effects of symmetry breaking and symmetry elevation. Phys. Rev. B 81, 161307(R) (2010).

18. Besombes, L., Kheng, K. \& Martrou, D. Exciton and biexciton fine structure in single elongated islands grown on a vicinal surface. Phys. Rev. Lett. 85, $425-428$ (2000).

19. Belhadj, T. et al. Impact of heavy hole-light hole coupling on optical selection rules in GaAs quantum dots. Appl. Phys. Lett. 97, 051111 (2010).

20. Li, L. et al. Control of polarization and dipole moment in low-dimensional semiconductor nanostructures. Appl. Phys. Lett. 95, 221116 (2009).

21. Ridha, P. et al. Polarization properties of columnar quantum dots: Effects of aspect ratio and compositional contrast. IEEE J. Quant. Electron. 46, 197-204 (2010).

22. Troncale, V., Karlsson, K. F., Pelucchi, E., Rudra, A. \& Kapon, E. Control of valence band states in pyramidal quantum dot-in-dot semiconductor heterostructures. Appl. Phys. Lett. 91, 241909 (2007).

23. Huo, Y. H., Rastelli, A. \& Schmidt, O. G. Ultra-small excitonic fine structure splitting in highly symmetric quantum dots on GaAs(001) substrate. Appl. Phys. Lett. 102, 152105 (2013).

24. Owen, D. L., Lackner, D., Pitts, O. J., Watkins, S. P. \& Mooney, P. M. In-place bonding of $\mathrm{GaAs} / \mathrm{InGaAs} / \mathrm{GaAs}$ heterostructures to $\mathrm{GaAs}(001)$. Semicond. Sci. Technol. 24, 035011 (2009).

25. Zander, T. et al. Epitaxial quantum dots in stretchable optical microcavities. Opt. Express 17, 22452-22461 (2009).

26. Ding, F. et al. Tuning the exciton binding energies in single self-assembled InGaAs/GaAs quantum dots by piezoelectric-induced biaxial stress. Phys. Rev. Lett. 104, 067405 (2010).

27. Bir, G. L. \& Pikus, G. E. Symmetry and Strain-induced Effects in Semiconductors (Wiley, 1974).

28. Meier, F. \& Zakharchenva, B. P. (eds) Optical Orientation (Modern Physics in Condensed Matter Science, Vol 8, Elsevier Science, 1984).

29. Bayer, M. et al. Fine structure of neutral and charged excitons in self-assembled In(Ga)As/(Al)GaAs quantum dots. Phys. Rev. B 65, 195315 (2002). 
30. Witek, B. J. et al. Measurement of the g-factor tensor in a quantum dot and disentanglement of exciton spins. Phys. Rev. B 84, 195305 (2011).

31. Bester, G., Nair, S. \& Zunger, A. Pseudopotential calculation of the excitonic fine structure of million-atom self-assembled $\operatorname{In}_{1-x} \mathrm{Ga}_{x} \mathrm{As} / \mathrm{GaAs}$ quantum dots. Phys. Rev. B 67, 161306(R) (2003).

32. Bester, G. Electronic excitations in nanostructures: An empirical pseudopotential based approach. J. Phys. Condens. Matter 21, 023202 (2009).

33. Rastelli, A. et al. Hierarchical self-assembly of GaAs/AlGaAs Quantum dots. Phys. Rev. Lett. 92, 166104 (2004).

34. Tonin, C. et al. Polarization properties of excitonic qubits in single self-assembled quantum dots. Phys. Rev. B. 85, 155303 (2012).

35. Trotta, R. et al. Nanomembrane quantum-light-emitting diodes integrated onto piezoelectric actuators. Adv. Mater. 24, 2668-2672 (2012).

\section{Acknowledgements}

We acknowledge P. Atkinson, Ch. Deneke, D. J. Thurmer and R. Engelhard for assistance with the molecular beam epitaxy, D. Grimm, B. Martin and S. Harazim for assistance in clean room maintenance, and G. Katsaros and R. Rezaev for fruitful discussions.

This work was financially supported by the BMBF project QuaHL-Rep (Contracts no 01BQ1032 and 01BQ1034), the DFG FOR730, the FOM (VIDI Grant), the European
Union Seventh Framework Programme 209 (FP7/2007-2013) under Grant Agreement No. 601126210 (HANAS) and the ERANET project QOptInt.

\section{Author contributions}

Y.H.H. grew and processed samples, measured micro-photoluminescence, and analysed data supported by S.K., J.X.Z., E.Z., R.T. and F.D., under supervision of A.R. and O.G.S. B.J.W. carried out micro-photoluminescence in magnetic field supported by N.A. and V.Z. and provided insightful interpretation of the experimental and theoretical results. J.R.C., R.S. and G.B. performed pseudopotential calculations and developed the mesoscopic model. R.G., D.K. and J.S. performed X-ray diffraction measurements. All authors discussed the results and contributed to the manuscript. A.R. conceived and coordinated the project, triggered by V.Z. and N.A.

\section{Additional information}

Supplementary information is available in the online version of the paper. Reprints and permissions information is available online at www.nature.com/reprints. Correspondence and requests for materials should be addressed to A.R.

\section{Competing financial interests}

The authors declare no competing financial interests. 